\title{
Les anticorps et scaffold bispéciftiques, des médicaments \\ innovants \\ en oncologie impliquant le ciblage des cellules immunitaires
}

$>$ Une connaissance approfondie de la biologie et de l'immunologie des tumeurs, mais aussi la conception de formats innovants d'anticorps et d'autres charpentes (ou scaffolds) protéiques ont permis de générer une véritable explosion de nouvelles molécules à visée thérapeutique au cours de ces 10 dernières années. Dans ce cadre, les anticorps bispécifiques (Abs) tiennent une place de choix. Ils permettent en effet, ( 1 ) d'apporter des propriétés biologiques et pharmacologiques nouvelles qui dépendent de l'engagement simultané des deux cibles, (2) d'améliorer le profil de sécurité par rapport à une combinaison d'anticorps en favorisant sa localisation tumorale en oncologie, et également (3) de combiner en une seule molécule les activités de deux anticorps conventionnels, réduisant ainsi les coûts de développement clinique et de fabrication. Cet article de revue a pour objectif d'analyser les différentes molécules bispécifiques décrites à ce jour dans le domaine de l'immuno-oncologie, et de présenter leurs différents formats et principales propriétés. <

Une révolution dans le traitement des cancers : le ciblage des cellules immunitaires

Ces dernières années ont vu une révolution s'opérer dans les traitements des cancers avec l'avènement des immunothérapies, et plus particulièrement des anticorps inhibiteurs de points de contrôle immunitaire (immune checkpoint inhibitors). Ainsi, les récents essais cliniques ont permis d'identifier plus de 30 types de cancer sensibles aux traitements anti-PD-1 (programmed cell death-1)/PD-Ll (programmed cell death-ligand 1) [1]. C'est la première fois en oncologie qu'une classe de médicaments démontre un si large spectre d'activités en monothérapie. Cependant, de nombreux effets secondaires limitent parfois l'usage de ces thérapies. De plus, une majorité de patients sont malheureusement réfractaires à ce type d'approche,

Patrick Chames $^{1}$, Thierry Wurch ${ }^{2}$

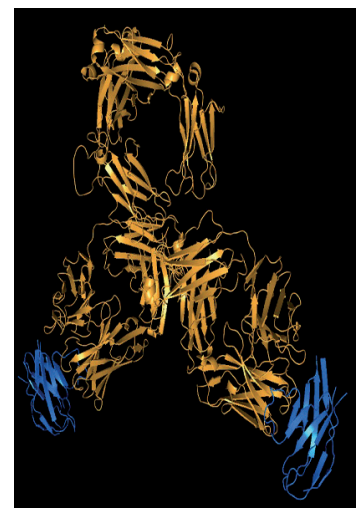

${ }^{1}$ Aix Marseille Univ, CNRS, Inserm, Institut Paoli-Calmettes, CRCM, Marseille, France.

${ }^{2}$ Centre d'Innovation Thérapeutique en Oncologie Servier, F78290 Croissy-sur-Seine, France.

thierry.wurch@servier.com

particulièrement ceux dont les tumeurs sont faiblement infiltrées par les cellules effectrices du système immunitaire tels que lymphocytes $T$ (LT) et cellules NK (natural killer) [2]. Pour pallier ce problème, plusieurs approches d'immunothérapie sont activement explorées. Deux approches parmi les plus prometteuses semblent être les thérapies cellulaires utilisant des cellules modifiées génétiquement (comme les CAR-T cells), et l'utilisation d'anticorps bispécifiques (Abs). II y a, à ce jour, plus de 60 Abs (ou apparentés) en développement clinique, dont $80 \%$ fonctionnent en induisant une synapse entre une cellule tumorale et une cellule immunitaire. Quelques exemples de structures bispécifiques sont présentés dans la Figure 1. Le but de ces Abs est de recruter et d'activer les cellules effectrices au sein du microenvironnement tumoral dans le but de concentrer les effets pharmacologiques sur la zone tumorale pour améliorer l'efficacité thérapeutique de cette immunothérapie, tout en réduisant des effets indésirables liés à l'activation du système immunitaire en comparaison à une immunothérapie provoquant une immunomodulation systémique.

Dans cette revue, nous allons présenter les principales approches thérapeutiques impliquant des Abs dont le mécanisme d'action principal réside dans la réactivation des cellules du système immunitaire (LT, cellules NK), et décrire les différents formats protéiques mis en œuvre (reposant soit sur une structure d'immunoglobuline (Ig), soit sur des 


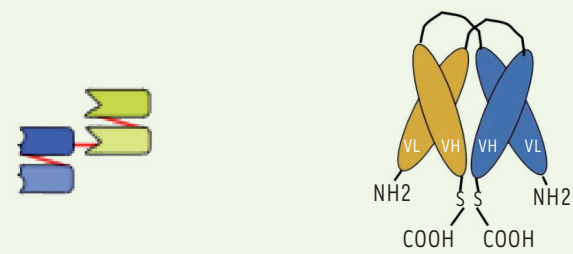

BiTદ®

DART ${ }^{\circledR}$
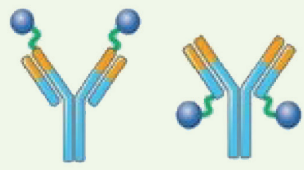

Exemples de protéines de fusion bispécifiques anticorps-anticaline ${ }^{\circledR}$

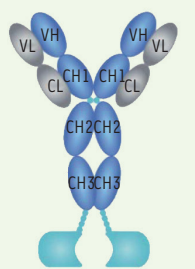

Protéine de fusion anticorpsleurre de TGF $\beta$

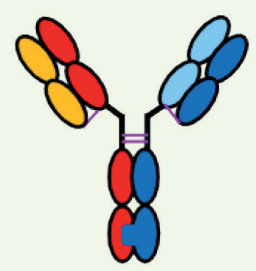

CrossMab ${ }^{\circledR}$

( $\mathrm{KiH})$

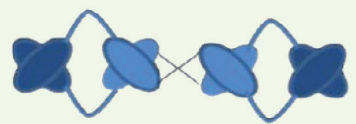

TandAb ${ }^{\circledR}$

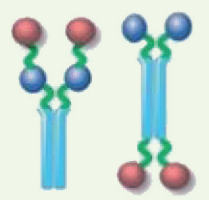

Exemples de protéines de fusion bispécifiques anticaline ${ }^{\circledR}-\mathrm{Fc}$

Figure 1. Quelques examples schématiques d'anticorps ou de scaffolds bispécifiques. Pour avoir des détails sur les structures de ces molécules, se référer aux références [3] et [4]. $\mathrm{KiH}$ : mutations « nœud dans la cavité » ou Knob-into-hole.

charpentes (ou scaffolds) protéiques non apparentées aux Ig. De par la flexibilité d'ingénierie de ce type de molécules bispécifiques, leurs champs d'applications vont bien au-delà du recrutement de cellules immununitaires; néanmoins, cette revue analysera uniquement les Abs ayant un mécanisme d'action immuno-modulateur et leurs applications en immunothérapie des cancers. De nombreux articles de revue décrivent de manière plus générale l'ingénierie et les applications des Abs dans les autres domaines, certains sont cités ci-après [3-5].

\section{Anticorps bispécifiques ciblant les lymphocytes T : BiTE, DART et autres formats}

Parmi les concepts d'immunomodulation, les thérapies ciblant les LT sont de loin les plus avancées, avec un produit approuvé et plusieurs composés en développement clinique. Les principales molécules sont résumées dans le Tableau I. Bien que les bénéfices cliniques les plus impressionnants soient pour l'instant obtenus sur des tumeurs hématologiques par nature plus accessibles, ces traitements pourraient en principe être aussi utilisés pour des tumeurs solides.
Les BiTE (bispecific T cell engagers) sont des Abs ciblant simultanément un antigène associé à la tumeur et le complexe récepteur des $\mathrm{LT}$ (ou TCR) via la chaîne $\mathrm{CD} 3 \varepsilon$, redirigeant ainsi la cytotoxicité des lymphocytes $T$ vers les cellules malignes, indépendamment de la spécificité de ces cellules. Une grande partie de la population de LT est donc activée polyclonalement. II faut souligner que l'activation des lymphocytes $T$ semble indépendante de la costimulation via CD28 ou via l'IL(interleukine)-2. Ceci pourrait s'expliquer par l'établissement d'une synapse immunologique étroite entre le lymphocyte $T$ et la cellule tumorale via l'Abs, agrégeant ainsi les complexes TCR/ CD3 tout en excluant la phosphatase CD45 plus volumineuse, qui joue un rôle immunomodulateur négatif [6]. Une autre hypothèse suggère que les cellules effectrices principalement responsables de l'effet cytotoxique seraient des lymphocytes T mémoires, nécessitant moins de co-stimulation pour être complètement activés [7]. L'activation des LT peut être obtenue à de très faibles 


\begin{tabular}{|c|c|c|c|c|}
\hline $\begin{array}{l}\text { Mécanisme } \\
\text { d'action }\end{array}$ & Format & Cibles & Molécule & $\begin{array}{c}\text { Stade de } \\
\text { développement }\end{array}$ \\
\hline \multirow{11}{*}{$\begin{array}{l}\text { Reciblage des LT } \\
\text { cytotoxiques }\end{array}$} & BiTE & CD19xCD3 & Blinatumomab & Approuvé \\
\hline & Knob-into-hole & $\mathrm{CD} 20 \times \mathrm{CD} 3$ & XmAb13676 & Ph. I \\
\hline & Knob-into-hole & $\mathrm{CD} 20 \times \mathrm{CD} 3$ & Mosunétuzumab & Ph. I \\
\hline & $2: 1 \mathrm{TCB}$ & $\mathrm{CD} 20 \times \mathrm{CD} 3$ & CD20-TCB & Ph. I \\
\hline & Knob-into-hole & CD123xCD3 & Xmabl4045 & Ph. I \\
\hline & DART & $\mathrm{CD} 123 \times \mathrm{CD} 3$ & Flotétuzumab & Ph. I \\
\hline & Duobody & CD123xCD3 & JNJ-63709178 & Ph. I \\
\hline & BiTદ & BCMAxCD3 & BI 836909 & Ph. I \\
\hline & Duobody & BCMAxCD3 & JNJ-64007957 & Ph. I \\
\hline & 2:1 TCB & $C E A x C D 3$ & CEA-TCB & Ph. I \\
\hline & DART & $\mathrm{B} 7 \mathrm{H} 3 \times \mathrm{CD} 3$ & MGD009 & Ph. I \\
\hline \multirow{2}{*}{ Reciblage des LT $\gamma \delta$} & Tandem de Nb & $\varepsilon G F R x \delta 2$ & N.A. & PC \\
\hline & Tandem de Nb & HER2/neuxy9 & N.A. & PC \\
\hline \multirow{6}{*}{$\begin{array}{l}\text { Reciblage des cellules } \\
\text { NK }\end{array}$} & Tandab & $\mathrm{CD} 30 \times \mathrm{CD} 16$ & AFM13 & Ph. II \\
\hline & Tandab & BCMAxCD 16 & AFM26 & PC \\
\hline & trike & $\mathrm{CD} 133 \times \mathrm{CD} 16$ & N.A. & PC \\
\hline & aTriflex & BCMAxCD $200 \times C D 16$ & N.A. & PC \\
\hline & BiKE & CSIXNKG2D & N.A. & PC \\
\hline & IgG-like & CD20xNKp46 & - & PC \\
\hline
\end{tabular}

Tableau I. Anticorps bispécifiques activateurs des cellules immunes cytotoxiques. PC : préclinique ; Ph. 1 : phase clinique 1 ; Ph. II : phase clinique II.

doses d'anticorps ou contre des antigènes tumoraux à faible niveau d'expression, de l'ordre de quelques centaines à quelques milliers de molécules par cellule. Le concept de reciblage des LT est exploré depuis de nombreuses années et des effets cliniques favorables ont été mis en évidence. Cependant, peu de thérapies de reciblage des LT ont été commercialisées ou ont atteint un stade de développement clinique avancé, probablement en raison d'une toxicité importante, de problèmes de fabrication, d'une immunogénicité et de faibles taux de réponse dans les tumeurs solides. Nous ne parlerons pas ici du catumaxomab, premier Abs anti-CD3 approuvé en 2009 et ciblant l'antigène tumoral EpCAM (epithelial cell adhesion molecule), mais retiré du marché en 2017.

\section{Le blinatumomab}

Le blinatumomab (Blincyto ${ }^{\circledR}$ ) est le seul anticorps bispécifique actuellement approuvé. II est constitué d'un fragment variable simple chaîne Fv (scFv) d'un anticorps murin anti-CD19 fusionné à un scFv murin anti-CD3 via un court peptide de liaison (GGGGS). En 2004, un essai en escalade de dose de phase I par administration en perfusion continue a débuté, conduisant à des réponses cliniques significatives à une dose de $15 \mu \mathrm{g} / \mathrm{m}^{2} /$ jour. Dix ans plus tard, le blinatumomab était approuvé par la food and drug administration (FDA) américaine pour le traitement de la leucémie aiguë lymphoblastique, en rechute et réfractaire [8]. L'approbation accélérée et conditionnelle par la FDA en 2014 a depuis été convertie en une approbation définitive en juillet 2017 , incluant également les patients pédiatriques et Philadelphia-positifs (présentant une fusion de gènes $B C R-A B L 1$ ). Un inconvénient majeur de cet Abs reste sa courte demi-vie, dûe à l'absence de région constante $\mathrm{Fc}$; ce format nécessite une administration intraveineuse continue par perfusion grâce à un système portatif sur plusieurs semaines. Cependant, la courte demi-vie peut avoir certains avantages. Les effets secondaires graves sont ainsi gérés par arrêt de la perfusion et sont généralement réversibles en quelques heures. La possibilité d'administrer le blinatumomab par voie sous-cutanée est actuellement testée dans un essai de phase lb (NCT02961881). Le blinatumomab est évalué dans un essai clinique pour le traitement des lymphomes non-hodgkiniens ( $\mathrm{NHL}$ ). Parmi les patients traités à la dose cible de $60 \mu \mathrm{g}$ / $\mathrm{m}^{2} /$ jour lors d'un essai de phase I $(\mathrm{n}=35)$, le taux de réponse global était de $69 \%$ pour tous les sous-types de NHL, associé à des taux de réponses complètes de l'ordre de $40 \%$. Une étude de phase II a cependant mis en évidence des problèmes de neurotoxicité et de type syndrome de libération de cytokines, limitant la quantité injectable [9].

\section{Les autres Abs anti-CD3 en essai clinique précoce}

Suite au succès de ce premier anticorps bispécifique ciblant la molécule CD3, de nombreuses alternatives ont vu le jour, tant en terme d'antigène ciblé que de format proposé. 


\section{CD20}

Au moins trois molécules bispécifiques en essai clinique ciblent CD20, un antigène retrouvé sur les lymphocytes $B$ et notamment ciblé par l'anticorps rituximab. Le XmAbl3676 possède une région Fc modifiée améliorant sa liaison aux cellules NK et aux macrophages ainsi que sa demi-vie sérique. Cette molécule utilise une région $\mathrm{Fc}$ de type «nœud dans la cavité » (knob-into-hole, $\mathrm{KIH})^{1}$, fusionné à un fragment Fab anti-CD20 et à un scFv anti-CD3. Cette molécule est actuellement testée depuis 2017 contre le NHL (NCT02924402). Le mosunétuzumab (BTCT4465A, RG7828) est un anticorps entier très similaire à une IgGl humaine, dont la structure repose aussi sur l'approche KIH. II est actuellement testé en tant qu'agent unique ou associé à l'atézolizumab chez des patients atteints de NHL ou de leucémie lymphoïde chronique (LLC) (NCT02500407), ou pour le traitement du lymphome $B$ diffus à grandes cellules (NCT03677154). CD20-TCB (R07082859) est un anticorps actuellement testé en essai clinique de phase I contre le lymphome à grandes cellules B diffus (NCT03467373), le lymphome folliculaire (NCT03467373) et le NHL (NCT03075696). Son format très particulier (2:1 CD20-TCB) est constitué de deux Fab anti-CD20 et d'un Fab anti-CD3E, I'un des Fab anti-CD20 étant directement fusionné au Fab anti-CD3\& via un peptide de liaison flexible [10].

\section{CD123}

CD123 est la chaîne alpha du récepteur de l'interleukine-3 humaine (IL-3R $\alpha$ ). II favorise la prolifération et la différenciation des cellules hématopoïétiques. CD123 est fortement exprimé sur les blastes et les cellules souches leucémiques (LSC) de leucémie myéloïde aiguë (LMA) et est associé à un mauvais pronostic chez les patients qui en sont atteints. C'est donc une cible attrayante pour recruter des lymphocytes T contre les blastes et les LSC [11]. Xmab14045 est un anticorps

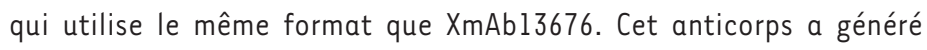
de très bons résultats précliniques [12] et est testé depuis 2016 en essai clinique pour le traitement de la LMA et d'autres hémopathies malignes exprimant CD123 (NCT02730312).

Le flotetuzumab) est fondé sur un format sans région $\mathrm{Fc}$, nommé DART (dual-affinity re-targeting). Ce format correspond au format diabody ${ }^{2}$ stabilisé par un pont disulfure. Des résultats précliniques prometteurs [13] ont conduit à des essais cliniques chez des patients atteints de LMA en rechute ou réfractaires, en cours depuis 2014 (NCT02152956, NCT03739606).

JNJ-63709178 est un Abs humanisé de type IgG4 dont la région Fc est silencieuse, fondée sur la technologie duobody. Suite à des résultats précliniques là aussi très encourageants [14], cet anticorps est testé en clinique contre les LMA en rechute ou réfractaires, depuis 2016 (NCT02715011).

\footnotetext{
${ }^{1}$ Technique qui permet d'améliorer l'association entre régions Fc des chaînes lourdes en modifiant des acides aminés des domaines $\mathrm{CH} 3$.

${ }^{2}$ Un diabody est un dimère non covalent formé de deux scFv dont le peptide de liaison entre les domaines VH et VL est trop court pour permettre un appariement VH/VL d'une même polypeptide, conduisant ainsi à des interactions VH/VL entre deux peptides. Le format DART possède de plus une cystéine C-terminale conduisant à la stabilisation du dimère ainsi formé par un pont disulfure.
}

\section{BCMA}

BCMA ( $B$-cell maturation antigen) est un récepteur très spécifique des plasmocytes. Il joue un rôle central dans la régulation de la maturation et de la différenciation des lymphocytes B en plasmocytes. BCMA est exprimé sur les plasmocytes de patients atteints de myélome multiple (MM) ainsi que sur les cellules dendritiques plasmacytoïdes, favorisant la croissance, la survie et la résistance des cellules MM. N'étant pas exprimé sur les lymphocytes B naïs et la plupart des cellules mémoires, sur les cellules hématopoïétiques ou toute autre cellule tissulaire normale, il constitue donc une cible idéale pour les thérapies de reciblage des LT [15].

BI 836909 est un anticorps bispécifique de type BiTદ. Suite à des résultats précliniques très favorables [16], ce BiTદ est actuellement testé dans des essais cliniques de phase I contre le MM (NCT02514239). JNJ-64007957 est un autre Abs au format duobody en phase clinique I depuis 2017 contre les MM récidivant ou réfractaire (NCT03145181).

D'autres antigènes sont ciblés par des Abs. On pourra notamment retenir l'ACE (antigène carcino-embryonnaire) ciblé par CEA-TCB (R06958688), utilisant le format 2:1 TCB décrit plus haut, qui a récemment conduit à des résultats cliniques dans le cancer colorectal métastatique résistant à la chimiothérapie, incluant des cancers colorectaux métastatiques à microsatellites stables (NCT02650713, NCT02324257). Également prometteur, l'Abs au format DART (MGD009) ciblant B7H3, un récepteur de la famille des molécules B7 impliqué dans la régulation immunitaire, et surexprimé dans un large éventail de cellules cancéreuses, y compris les cellules souches cancéreuses, ainsi que sur les cellules endothliales du système vasculaire de la tumeur. Ce DART était testé depuis 2015 dans un essai clinique de phase I (NCT02628535). Cependant, cette étude a été arrêtée en décembre 2018 suite à une toxicité hépatique observée chez certains patients.

\section{Vy9V反2 T-cell engagers: vers une nouvelle génération de BiT\&?}

Les $L T \gamma \delta$ constituent une sous-population représentant 1 à $10 \%$ des leucocytes. Leur activation via leur TCR est indépendante du complexe majeur d'histocompatibilité $(\mathrm{CMH})$ et conduit à la libération de cytokines et à une cytotoxicité. Les lymphocytes $\mathrm{T} V \gamma 9 \mathrm{~V} \delta 2$ constituent la principale population de cellules $T \gamma \delta$ et leur infiltration tumorale a été associée à un facteur de bon pronostic dans divers types de tumeurs [17]. Leur ciblage thérapeutique paraît donc pertinent. En 2017, de Bruin et al. ont généré un anticorps bispécifique de format original fondé sur l'utilisation d'anticorps à domaine unique dérivés d'anticorps de lama (aussi appelé nanobodies) anti-EGFR 
(epidermal growth factor receptor) et anti-chaîne $\delta 2$ du TCR, fusionnés en tandem. Cette molécule a conduit à des résultats précliniques intéressants incluant une forte activation des cellules $T$ V $\gamma 9 V \delta 2$ et la lyse subséquente des cellules tumorales in vitro et in vivo dans un modèle de xénogreffe, de façon indépendante à la présence des mutations tumorales KRAS et BRAF [18]. De façon similaire, Oberg et al. ont pu démontrer l'efficacité du reciblage des $L T \gamma \delta$ grâce un Abs anti-HER2 $\times \gamma 9$ dans un modèle de transfert adoptif de lymphocytes $T \gamma \delta$ chez des souris immunodéprimées (souris (severe combined immunodeficiency) SCID-Beige) ayant reçu une xénogreffe de tumeur pancréatique [18].

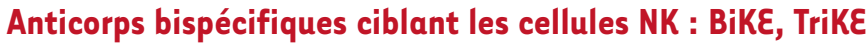 et plus}

Une alternative à l'utilisation du potentiel cytotoxique des lymphocytes $T$ pour l'éradication de la tumeur consiste à rediriger et activer la fonction cytotoxique des cellules NK vers les cellules malignes. Ces cellules ont aussi un potentiel thérapeutique important. En effet, en plus de pouvoir reconnaître de façon précoce les transformations des cellules tumorales, celles-ci sont capables de sécréter un ensemble de cytokines et de chimiokines dont le rôle est d'activer et de recruter les autres acteurs du système immunitaire, plus particulièrement les cellules dendritiques capables d'effectuer une présentation croisée («cross-presentation ») via les complexes CMH de classe I, des antigène tumoraux libérés par la lyse des cellules tumorales [19]. Plusieurs récepteurs capables d'activer la fonction cytotoxique des cellules NK ont été décrits, notamment CD16, NKp30, NKp46, NKG2D (natural killer group 2, member D) et DNAM-1 (DNAX accessory molecule-1). CD16a (le récepteur de la région $\mathrm{Fc}$ de type III, ou RFcyllla), dont l'engagment par des IgG complexées à leur antigène cible conduit à l'activation des cellules NK sans besoin d'un second signal, et qui est également exprimé sur les macrophages et les lymphocytes $T \gamma \delta$, est, à ce jour, la cible privilégiée des Abs ciblant les cellules NK, appelés BiKE pour bispecific killer cell engagers.

\section{Les « NK engagers 》 ciblant CD16a}

Le format le plus utilisé pour générer des BikE est le TandAb (pour tandem diabody), un format fondé sur le principe des diabodies, c'està-dire des fusions VH-VL utilisant des peptides de liaison suffisamment courts pour imposer une dimérisation en trans. Les chaînes des TandAb comportent 4 domaines, conduisant après dimérisation à des molécules bispécifiques tétravalentes de plus de 100 kDa. La molécule la plus étudiée est le TandAb AFM13 [20]. Cette molécule cible CD30, un membre de la superfamille des récepteurs du facteur de nécrose tumorale (TNFRSF), typiquement exprimé dans certaines hémopathies malignes, dont, notamment, le lymphome anaplasique à grandes cellules et le lymphome de Hodgkin. Une particularité de la molécule AFM13 est de pouvoir lier CD16a, exprimé notamment par les cellules NK et les macrophages, sans pour autant interagir avec le CD16b exprimé par les neutrophiles. Elle est ainsi capable de stimuler l'ADCC des cellules NK contre des cellules tumorales exprimant CD30. Lors d'une étude de phase I portant sur le lymphome de Hodgkin réfrac- taire, AFM13 a entraîné une activation significative des cellules NK et une diminution de CD30 soluble dans le sang périphérique, sans provoquer d'effet secondaire important [21]. Cette molécule est actuellement testée dans deux essais de phase II contre les lymphomes exprimant CD30 (NCT02321592, NCT03192202). Utilisant le même format mais ciblant cette fois BCMA, AFM26 est en phase préclinique contre le myélome multiple [22]. Le TandAb AFM24, qui cible l'EGFR dans les tumeurs solides, a, quant à lui, démontré une efficacité in vivo dans un modèle de souris humanisée hu-NOG (souris NOD/SCID/IL2R $\gamma^{\text {null }}$ ), tout en présentant un profil d'innocuité favorable chez les singes cynomolgus [23].

\section{Les formats Trike et aTriFlex}

D'autres formats plus récents semblent être encore plus prometteurs. Les TrikE (trispecific NK cell engager) sont constitués de deux scFv fusionnés en $\mathrm{N}$ et $\mathrm{C}$-terminal à I'IL-15. Ce format permet d'activer les cellules NK via l'engagement du CDI6a, mais aussi de provoquer leur prolifération et une survie prolongée grâce à l'action ciblée de l'IL-15, ce qui n'est pas le cas du format BiKE. Des résultats précliniques très prometteurs ont ainsi été obtenus avec un Trike ciblant CD133, un marqueur identifié comme étant fréquemment exprimé par les cellules souches cancéreuses de différents types de tumeurs [24]. Le format aTriflex utilise le concept du double ciblage, c'est-à-dire le ciblage simultané de deux antigènes de surface présentant une faible expression sur les cellules normales mais co-exprimés par une tumeur, en utilisant des affinités modérées pour favoriser la spécificité tumorale par un effet de gain d'avidité. Une preuve de concept a ainsi été établie avec un format trispécifique tétravalent présentant un site de liaison pour BCMA et CD200 et deux sites de liaison pour CD16a [25].

\section{Les $N K$ cell engagers ciblant d'autres récepteurs activateurs}

Finalement, de récents travaux suggèrent la possibilité de recibler les cellules NK par des récepteurs activateurs autres que CD16. Ainsi, le format BiKE a été utilisé pour cibler simultanément CS1 (ou CD319/SLAMF7), une molécule d'activation lymphocytaire de surface notamment surexprimée par les cellules de myélomes multiples, et NKG2D, un des récepteurs activateurs des cellules NK, des lymphocytes $T \mathrm{CD}^{+}, \mathrm{T} \gamma \delta$ et des cellules NKT. Des résultats précliniques in vitro et in vivo suggèrent ainsi que l'activation de plusieurs types de cellules effectrices immunitaires via NKG2D permet d'améliorer la cytotoxicité anti-tumorale [26].

NKp46 (ou NCRl) appartient à la famille des récepteurs de cytotoxicité naturelle (NCR). Il est considéré comme 


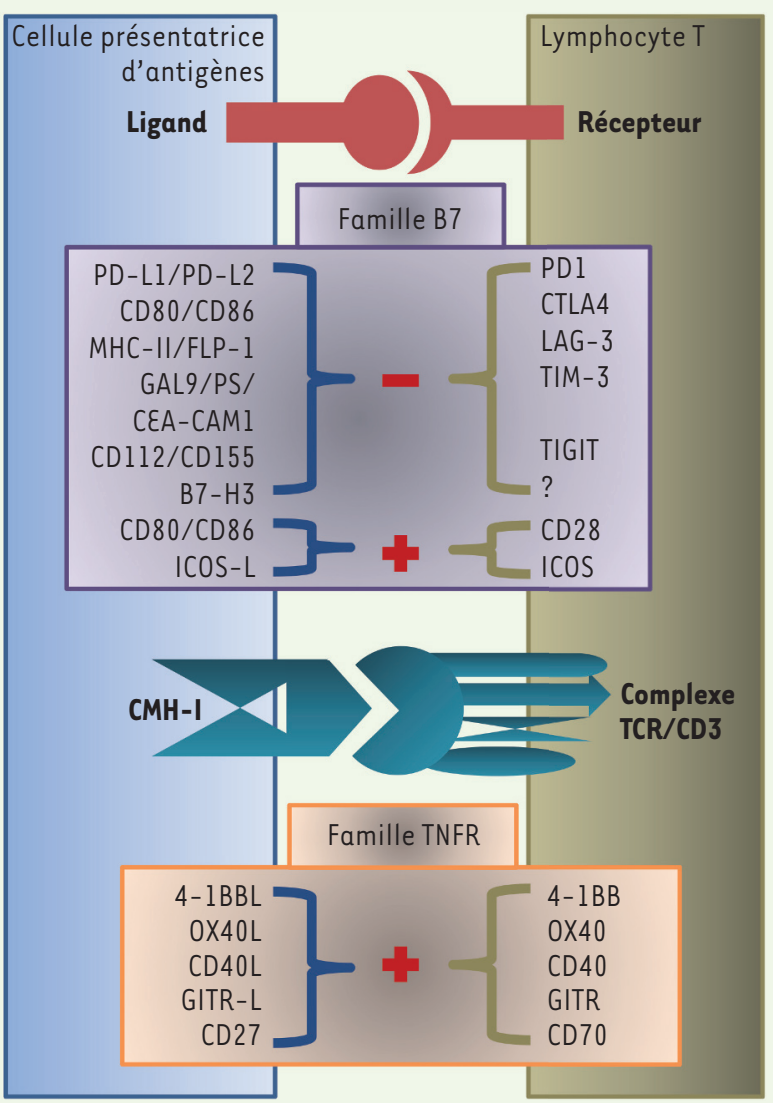

Figure 2. De nombreux récepteurs activateurs et inhibiteurs régulent la réponse des lymphocytes $T$. PD-1 : programmed cell death protein 1; CTLA-4 : cytolytic T-lymphocyte-associated protein 4; LAG-3: lymphocyte activation gene 3; TIM-3 : T cell immunoglobulin and mucin domain-containing-3; TIGIT : T-cell immunoreceptor with Ig and ITIM domains; ICOS : inducible T-cell costimulatory; GITR: glucocorticoid-induced tumor necrosis factor-related protein ; TCR : - cell receptor; CMH : complexe majeur d'histocompatibilité ; FLP-1 : fibrinogen-like protein 1; GAL9: galectine 9; PS: phosphatidylsérine; CEACAMl : carcinoembryonic antigen-related cell adhesion molecule 1 ; L : ligand.

un récepteur majeur activant les cellules NK, et est impliqué dans l'élimination des cellules cibles [27]. Il a été montré récemment in vitro qu'il était possible de recibler des cellules NK vers des cellules tumorales Daudi exprimant CD20, grâce à un Abs CD20 x NKp46 de façon plus efficace que l'anticorps anti-CD20 obinutuzumab. Dans un modèle de souris CB17 SCID greffées avec des cellules Raji, cette molécule a, là aussi, surpassé les résultats de survie obtenue avec l'obinutuzumab.

\section{Anticorps bispécifiques ciblant les points de contrôle immunitaire}

Depuis l'énorme succès médical des anticorps ciblant l'axe PD-1 (programmed cell death protein 1)/PD-Ll(programmed cell deathligand 1 ), un grand nombre d'essais cliniques combinant ces inhi- biteurs à d'autres anticorps anti-points de contrôle immunitaire (ICP), inhibiteurs ou co-stimulateurs, ont été initiés, parfois avec des résultats mitigés ou une toxicité accrue $[28,29]$. Ces résultats ont également ouvert la porte à la conception de nouveaux Abs combinant le ciblage de deux ICP inhibiteurs, ou le co-ciblage tumoral d'une protéine de co-stimulation, ou encore co-ciblant une cytokine.

\section{Co-ciblage d'immunomodulateurs co-inhibiteurs}

\section{Inhibition de l'axe PD-1/PD-L1}

Le ciblage de PD-1 ou PD-Ll a été combiné à celui d'autres ICP inhibiteurs, comme LAG-3 (lymphocyte activation gene 3 ), TIM-3 ( $T$ cell immunoglobulin and mucin domain-containing protein 3) ou CTLA-4 (cytolytic T-lymphocyte-associated protein 4), en utilisant des formats bispécifiques différents. La régulation des LT par les différents récepteurs inhibiteurs et co-activateurs est schématisée dans la Figure 2. Les Abs sont présentés dans le Tableau II.

La structure de la molécule MGD013 est fondée sur le format propriétaire DART ${ }^{\circledR}$. Mais, à la différence du flotétuzumab, le module DART ciblant PD-1 et LAG-3 possède une région $F_{c} d^{\prime} \lg G 4$ stabilisée dans sa région charnière $^{3}$. Cette molécule est ainsi bispécifique et bivalente pour ses 2 cibles et capable de les lier simultanément. MGD013 induit également une modulation de la signalisation intracellulaire induite par PD-1 et LAG-3 et stimule fortement la production d'interféron- $\gamma$ dans un modèle de stimulation de cellules mononucléées du sang périphérique (CMSP) par une entérotoxine staphylococcale. Son administration à des primates non humains a été bien tolérée jusqu'à une dose de $100 \mathrm{mg} / \mathrm{Kg} / \mathrm{semaine}^{4}$. Ces données encourageantes ont permis le démarrage d'une étude clinique chez des patients atteints de tumeurs solides métastatiques (NCT03219268).

La structure de la molécule FS118 est fondée sur le format Fcab ${ }^{\circledR}[30]$. II s'agit d'un anticorps ciblant PD-L1 auquel a été greffé au niveau de sa région Fc un nouveau domaine de liaison ciblant LAG-3. Le module de liaison $L A G-3$ a été sélectionné à partir d'une série de mutants du domaine $\mathrm{CH} 3$ présentant une forte liaison à la cible et une puissance de l'ordre du nanomolaire dans un test d'activation de lymphocytes T et d'inhibition de liaison du ligand, les molécules du CMH-II [31]. L'addition d'un site de liaison LAG-3 à un anticorps

\footnotetext{
${ }^{3}$ Une mutation Sérine228 en Proline dans la région charnière d'un anticorps d'isotype IgG4 permet d'éliminer la formation de demi-anticorps par le phénomène d'échange de fragments Fab appellé "Fab arm exchange".

${ }^{4}$ La Motte-Mohs et al., Society for Immunotherapy of Cancer (SITC) 2017.
} 


\begin{tabular}{|c|c|c|c|c|}
\hline Classe & Mécanisme d'action & Cibles & Molécules & $\begin{array}{c}\text { Stade de } \\
\text { développement }\end{array}$ \\
\hline $\begin{array}{l}\text { Immunomodulateurs } \\
\text { co-inhibiteurs }\end{array}$ & $\begin{array}{l}\text { Co-engagement de deux ICP } \\
\text { co-inhibiteurs induisant une } \\
\text { réactivation puissante des LT }\end{array}$ & $\begin{array}{l}\text { PD- } 1 \times \text { LAG-3 } \\
\text { PD- } 1 \times \text { TIM-3 } \\
\text { PD- } 1 \times \text { TIM-3 } \\
\text { PD-L1xLAG-3 } \\
\text { CTLA-4xLAG-3 } \\
\text { CTLA-4xPD 1 } \\
\text { CTLA-4xPD 1 }\end{array}$ & $\begin{array}{c}\text { MGD013 } \\
\text { R07121661 } \\
\text { MCLA-134 } \\
\text { FS118 } \\
\text { XmAb22841 } \\
\text { XmAb20717 } \\
\text { MGD019 }\end{array}$ & $\begin{array}{l}\text { Ph. I } \\
\text { Ph. I } \\
\text { PC } \\
\text { Ph. I } \\
\text { Ph.l } \\
\text { Ph. I } \\
\text { Ph. I }\end{array}$ \\
\hline $\begin{array}{l}\text { Immunomodulateurs } \\
\text { mixtes }\end{array}$ & $\begin{array}{l}\text { Co-ciblage de deux axes de régulation, } \\
\text { inhibiteur et co-stimulateur pour } \\
\text { maximiser l'activation des LT }\end{array}$ & $\begin{array}{l}\text { PD- } 1 \times I C O S \\
\text { PD-Llx4-1BB } \\
\text { PD-Llx4-1BB } \\
\text { PD-Llx4-1BB } \\
\text { PD-Llx4-1BB } \\
\text { PD-Llx4-1BB } \\
\text { PD-Llx4-1BB } \\
\text { CTLA-4x0X40 } \\
\text { CTLA-4xGITR }\end{array}$ & $\begin{array}{c}\text { XmAb23104 } \\
\text { PRS-344 } \\
\text { MCLA-145 } \\
\text { FS222 } \\
\text { ND021 } \\
- \\
- \\
\text { ATOR-1015 } \\
\text { ATOR-1144 }\end{array}$ & $\begin{array}{c}\text { Ph. I } \\
\text { PC } \\
\text { Ph.l } \\
\text { PC } \\
\text { PC } \\
\text { PC } \\
\text { PC } \\
\text { Ph.l } \\
\text { PC }\end{array}$ \\
\hline $\begin{array}{l}\text { Immunomodulateurs } \\
\text { tumeur-spécifique }\end{array}$ & $\begin{array}{l}\text { Ciblage sélectif de molécules de } \\
\text { co-stimulation exprimées par des } \\
\text { lymphocytes infiltrant les tumeurs } \\
\text { via la liaison à un antigène tumoral }\end{array}$ & $\begin{array}{l}\text { HER2/neux4-1BB } \\
\text { GPC } 3 \times 4-1 B B \\
5 T 4 \times 4-1 B B \\
\text { FAP } 44-1 B B \\
\text { FAPxCD } 40\end{array}$ & $\begin{array}{c}\text { PRS-343 } \\
\text { PRS-342 } \\
\text { AGL.APV-527 } \\
\text { MP310 } \\
-\end{array}$ & $\begin{array}{l}\text { Ph. I } \\
P C \\
P C \\
P C \\
P C\end{array}$ \\
\hline $\begin{array}{l}\text { Immunomodulateurs } \\
\text { et cytokines }\end{array}$ & $\begin{array}{l}\text { Ciblage d'un axe de régulation négatif } \\
\text { des LT associé à la séquestration d'une } \\
\text { cytokine pro-tumorale }\end{array}$ & $\begin{array}{l}\text { PD-Ll xTGF- } \beta \text { trap } \\
\text { CTLA- } 4 \text { xTGF- } \beta \text { trap }\end{array}$ & M7824 & $\begin{array}{l}\text { Ph. II } \\
\text { PC }\end{array}$ \\
\hline
\end{tabular}

Tableau II. Anticorps et scaffolds bispécifiques ciblant des points de contrôle immunitaire. PC : préclinique ; Ph. I : phase clinique I ; Ph. II : phase clinique II.

anti-PD-Ll permet à ce dernier d'induire une activation des LT CD $8^{+}$ supérieure à celle induite par l'anticorps anti-PD-Ll seul ${ }^{5}$. En utilisant un composé apparenté, capable de se lier aux cibles LAG-3 et PD-L1 de souris, des études de xénogreffes de tumeurs de côlon de souris MC38 à des animaux immunocompétents ont montré une activité anti-tumorale de l'Abs comparable à celle obtenue avec la combinaison anticorps anti-LAG-3/anti-PD-L1, associée à une modification du rapport

\footnotetext{
${ }^{5}$ Kraman et al., AACR 2018, Abstract 2719,
}

LT CD8/Treg dans l'infiltrat tumoral. Le composé FS118 est actuellement en essai clinique précoce chez des patients atteints de cancers et ayant progressé sous traitement ciblant l'axe PD-1/PD-LI (NCT03440437). À ce jour, les inhibiteurs de LAG-3 sont principalement évalués pour leur capacité à bloquer l'interaction avec son ligand, les molécules du CMH-II. Néanmoins, un second ligand de LAG-3 a été identifié très récemment, FLP-1 (fibrogen-like protein 1) [32]. II sera important 
de connaître la capacité des molécules existantes à inhiber l'action de ce nouveau ligand, cette découverte ouvrant aussi la porte à de nouvelles molécules bispécifiques au mécanisme d'action différent.

TIM-3 est une autre protéine de contrôle immunitaire combinée à un ciblage de PD-1. Bien que la biologie de cette cible soit plus complexe [33], son rôle dans l'échappement ou la résistance à un traitement par anticorps anti-PD-1 a été montré et deux molécules co-ciblant PD-1 et TIM-3 ont été produite (R07121661 et MCLA-134, voir Tableau II).

La conception de R07121661 est fondée sur le format CrossMab ${ }^{\circledR}$ de type [1+1] chargé VH-VL+/- $^{2}$ [34]: le domaine variable de l'un des bras d'anticorps possède une commutation («switch») CHl-CL et l'association VH-VL sur l'autre bras est favorisée par l'introduction de résidus chargés dans les domaines $\mathrm{CHl}-\mathrm{CL}$, permettant ainsi l'association spécifique de la chaîne légère avec la chaîne lourde appropriée, l'hétérodimérisation des chaînes lourdes étant réalisée grâce à I'introduction de mutations $\mathrm{KIH}$ [34]. Ce produit est actuellement en développement clinique précoce chez les patients atteints de tumeurs solides métastatiques (NCT03708328). Le second produit co-ciblant PD-1 et TIM-3, MCLA-134, est fondé sur la technologie Biclonics ${ }^{\circledR}$. À partir de deux anticorps sélectionnés par immunisation de souris $\mathrm{MeMo}^{6}$ et par ingénierie du domaine $\mathrm{CH} 3$ de leur région $\mathrm{Fc}$, des Abs sont produits par hétérodimérisation avec une grande efficacité et une production robuste.

\section{Inhibition de CTLA-4}

Le ciblage du point de contrôle immunitaire CTLA-4 a également été travaillé dans un contexte bispécifique de co-engagement avec un second ICP, tel que PD-1 ou LAG-3. Deux molécules de ce type, XmAb20717 et XmAb22841, sont fondées sur une bispécificité combinée à une technologie de modulation de la pharmacocinétique nommée Xtend ${ }^{\circledR}[35]$. Le format bispécifique mis en œuvre combine (1) I'utilisation d'un scFv en tant que l'un des domaines variables et d'un Fab et (2) l'ingénierie du domaine $\mathrm{CH} 3$ de la région $\mathrm{Fc}$ afin de favoriser l'association hétérodimérique des 2 chaînes lourdes par l'introduction de mutations ponctuelles sur chaque chaîne [36]. Dans les 2 situations, la molécule est bispécifique et monovalente pour chacune des cibles. Pour les deux molécules, une activité anti-tumorale significative est observée dans des modèles murins de co-greffes de cellules tumorales humaines et de cellules mononucléées du sang périphérique. La molécule XmAb20717 est actuellement en essai clinique précoce chez des patients atteints de tumeurs solides métastatiques (NCT03517488). Le format DART ${ }^{\circledR}$ a également été appliqué pour cibler CTLA-4 et PD-1. La molécule générée, MGD019, est constituée d'un DART fusionné à la région Fc d'une chaîne lourde d'IgG4 stabilisée au niveau de sa région charnière. Ce produit est ainsi bivalent et se lie simultanément aux deux cibles. II montre une puissance et efficacité comparable à la combinaison ipilimumab / nivolumab, mais est bien mieux toléré par les primates ${ }^{7}$. Ce produit est évalué chez des patients

${ }^{6}$ La souche MeMo de souris permet la génération d'anticorps composés d'une chaîne légère unique et donc identique pour tous les anticorps, facilitant ainsi l'obtention d'Abs.

${ }^{7}$ Berezhnoy et al., SITC 2017. atteints de tumeurs solides métastatiques dans le cadre d'un essai clinique précoce (NCT03761017).

\section{Ciblage d'immunomodulateurs co-inhibiteurs} et co-stimulateurs

Une seconde classe d'Abs immunomodulateurs ciblent simultanément un point de contrôle immunitaire inhibiteur et co-stimulateur. II s'agit dans ce cas de «lâcher le frein et appuyer sur l'accélérateur » pour faire une métaphore de conduite automobile. Même si cette image peut suggérer un possible emballement de la réponse immune, un tel co-ciblage permet au contraire de diriger l'action de co-stimulation au sein de la tumeur en ciblant, par exemple, PD-Ll. Ceci permettrait potentiellement de limiter les effets secondaires indésirables (inflammation du foie) observés chez des patients traités par l'urélumab [37].

Six molécules de ce type, co-ciblant PD-Ll et 4-1BB (ou tumor necrosis factor receptor superfamily member 9 , TNFRSF9) sont actuellement en développement préclinique démontrant le fort engouement de la communauté médico-scientifique pour cette approche. Elles ont toutes été présentées très récemment par la communauté scientifique. La première molécule, PRS-344/ ONC055 est construite par fusion d'un anticorps antiPD-Ll à une charpente protéique de type Anticalin ${ }^{\circledR}$, générant ainsi une molécule bispécifique et bivalente pour ses 2 spécificités. Cette molécule induit un signal de co-stimulation dépendant de l'expression de PD-Ll, mais conserve toutes les fonctionnalités de l'anticorps anti-PD-Ll parental. Son efficacité est comparable, voire supérieure à la combinaison des 2 composés parentaux dans des modèles pharmacologiques d'activation des LT induite soit par allo-réactivité soit par une entérotoxine staphylococcale ${ }^{8}$. La seconde molécule, MCLA-145 est conçue sur la base de la technologie Biclonics ${ }^{\circledR}$ identique à celle décrite précédemment pour MCLA-134. Au contraire de PRS-344, MCLA-145 est monovalent pour ses 2 cibles. La structure du composé FS222 est fondée sur un format Fcab similaire à celui du FS118. Un composé bispécifique PD-Llx4-1BB incorporant également un domaine de liaison à la sérum albumine humaine pour augmenter sa demi-vie, a été décrit également. Les technologies Duobody et DART ont été également utilisées pour co-cibler ces deux points de contrôle immunitaire. Le format duobody génère une molécule bispécifique et monovalente symétrique par hétéro-dimérisation des régions $\mathrm{Fc}$, alors que le format DART permet d'obtenir une molécule bispécifique, monovalente, mais asymétrique, ainsi qu'une variante

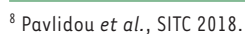


bispécifique, bivalente pour 4-1BB, monovalente pour PD-L1. Ces approches exploratoires permettent d'évaluer l'importance de paramètres de liaison telles l'affinité, la valence et la distance entre les deux modules de liaison permettant un engagement préférentiel des cibles en cis et/ou en trans.

Le composé XmAb23104 combine l'inhibition de CTLA-4 avec la co-stimulation de ICOS (inducible T cell costimulator) à partir du format bispécifique incorporant également la technologie Xtend d'augmentation de la demi-vie plasmatique (voir plus haut). Cette molécule permet un co-engagement des deux axes PD-1 et ICOS comme démontré par la supériorité de XmAb23104 comparé à chacun des produits parentaux dans des modèles d'activation de LT par la toxine staphylococcale?. La signature transcriptomique induite par XmAb23104 magnifie la réponse obtenue par la combinaison des anticorps anti-PD-l et antiICOS. Cette molécule est actuellement en développement clinique précoce chez des patients atteints de tumeurs solides métastatiques (étude DUET-3 - NCT03752398).

\section{Ciblage d'immunomodulateurs spécifique de tumeurs}

Une troisième classe d'Abs agit au niveau de point de contrôle de co-stimulation des LT infiltrant les tumeurs. Pour cela, la seconde spécificité cible un antigène tumoral, voire un antigène du microenvironnement. Les récepteurs de co-stimulation appartiennent principalement à la super-famille des récepteurs du TNF, comme par exemple 4-1BB, 0X40, GITR et CD40 (Figure 2) [38] ; ils s'activent par liaison de ligands trimériques. Ainsi, leur activation par des anticorps passe par la formation d'agrégats de récepteurs au niveau de la membrane des LT, dont la formation est favorisée par la densité antigénique de l'antigène tumoral ciblé. Un tel mécanisme d'action permettrait également de diminuer les effets indésirables tels que les dysfonctionnements hépatiques observés chez les patients traités par des anticorps agonistes de $4-1 B B$ et de CD40, en augmentant l'exposition tumorale des Abs.

Le prototype de ce nouveau mécanisme d'action est représenté par PRS-343. II s'agit d'un anticorps bispécifique issu de la fusion d'un anticalin ${ }^{10}$ dirigé contre 4-1BB en C-terminal de la chaîne lourde d'une version modifiée du trastuzumab (anticorps anti-HER2/neu) d'isotype IgG4. Cette molécule bispécifique et bivalente présente de bonnes propriétés physico-chimiques et une pharmacocinétique chez la souris comparable à celle d'anticorps conventionnels [39]. Dans un modèle de xénogreffe de cellules de tumeur mammaire humaine SKOV-3 et de CMSP, PRS-343 induit une forte régression de la tumeur associée à une augmentation de l'infiltration en cellules immunes $\mathrm{CD}_{4} 5^{+}$[39]. Ce composé est actuellement en essais cliniques précoces chez des patientes atteintes de tumeurs solides métastatiques soit en monothérapie (NCT03330561), soit en combinaison avec l'atézolizumab (NCT03650348). Fondé sur le même concept, un second projet ciblant l'antigène oncofœtal tumoral glypican-3 (GPC-3) associé au

\footnotetext{
${ }^{9}$ Moore et al., SITC 2017.

${ }^{10}$ Les anticalins sont des protéines artificielles dérivées des lipocalines, protéines naturelles de transport de petites molécules hydrophobes telles que les stéroides. Les anticalins représentent une alternative
} moléculaire de taille réduite aux anticorps. co-ciblage de 4-1BB est actuellement développé. À la différence de PRS-343, PRS-342 combine deux modules de liaison de type anticalin pour les deux spécificités ciblées reliés entre eux par une région Fc [39].

La molécule AGL.APV-527 est fondée sur un mécanisme d'action similaire à celui du PRS-343 mais cible un autre antigène oncofœtal tumoral, 5T4, toujours associé au point de contrôle co-stimulateur 4-1BB. Le format de cette molécule bispécifique et bivalente est ADAPTIR : il consiste à fusionner deux fragments simple-chaîne dérivés des deux anticorps parentaux à une région Fc dépourvue de fonctions effectrices. De par la forte expression de l'antigène 5T4 à la surface de nombreux types de cellules tumorales, l'agrégation de 4-1BB induite par la liaison de AGL.APV-527 au LT provoque leur activation et la sécrétion de cytokines. Ce mécanisme est associé à une forte inhibition de la croissance tumorale observée dans un modèle de xénogreffe de cellules tumorales de colon HCTI16 11 .

Le format DARPIN ${ }^{\circledR}$ a été utilisé pour le ciblage d'une protéine du microenvironnement tumoral liée à l'activation des fibroblastes (la FAP ou fibroblast activation protein). Ainsi MP310 cible 4-1BB et FAP sous forme d'une molécule bispécifique et monovalente, sans module d'extension de demi-vie. L'expression de FAP est nécessaire et suffisante pour induire l'activation des LT par engagement de MP310. Dans un modèle de xénogreffes de cellules tumorales HT-29, MP310 induit une forte régression de la croissance tumorale en combinaison avec une molécule engageant directement le récepteur des LT via CD $3^{12}$. Une seconde molécule a été conçue de manière similaire en engageant le point de contrôle de co-stimulation CD40 associé à l'expression de FAP.

\section{Association d'un immunomodulateur avec une cytokine}

Bien que l'association, sur une même molécule, d'un module d'immunomodulation et d'une cytokine soit très rationnelle [40], seuls deux exemples de ce type de composés existent dans la littérature, tous deux ayant pour but de bloquer l'action pro-tumorale du TGF- $\beta$ (tumor growth factor beta) [41] par l'utilisation d'un récepteur-leurre («decoy ») du récepteur de type 2 du TGF- $\beta$ (ou TGFBRII). Une première molécule, M7824, conçue par l'équipe de Jeffrey Schlom [National Cancer Institute $(\mathrm{NCl}$ )-National Institutes of Health $(\mathrm{NIH})$ ], utilise l'anticorps anti-PD-LI avelumab auquel est fusionnée en C-terminal de la chaîne lourde la séquence du domaine extracellulaire du TGFBRII. Ce composé s'associe simul-

\footnotetext{
${ }^{11}$ Fritzell et al., CIMT 2018.

12 Link et al., AACR2018.
} 
tanément au TGF $\beta 1$ et à PD-Ll et diminue la signalisation induite par ce ligand [42]. Dans des modèles syngéniques de tumeurs mammaires et de côlon, M7824 réduit fortement la croissance tumorale et augmente la survie des animaux traités via l'activation de $\mathrm{LT} C D 8^{+}$et des cellules NK [42], l'efficacité du composé étant supérieure à un traitement par monothérapie ciblant soit le TGF- $\beta$, soit PD-Ll. L'activité de ce composé chez l'homme est actuellement testée dans une dizaine d'essais cliniques soit en monothérapie, soit en combinaison avec de la chimiothérapie ou des agents immunomodulateurs. Les résultats précoces sur 19 patients d'une étude en monothérapie de recherche de dose et de toxicité montrent que le produit est toléré aux doses évaluées malgré l'apparition d'effets secondaires indésirables de grade supérieur ou égal à 3 chez quatre patients [43]. Un bénéfice clinique a été observé chez six patients, dont une réponse complète chez une patiente atteinte d'un cancer du col de l'utérus [43].

Utilisant le même concept, l'équipe de Atul Bedi de l'école de Médecine John Hopkins à Baltimore, a conçu un Abs à partir de la séquence de l'ipilimumab ciblant CTLA-4 fusionné en C-terminal de sa chaîne lourde au domaine extracellulaire de TGFBRII [44]. Cet Abs inhibe fortement l'infiltration de lymphocytes Treg et la différenciation des LT en Th17 dans un modèle de souris NSG (nod-scid gamma) humanisée avec des cellules souches hématopoïétiques humaines et greffées avec des cellules de lignées de mélanome A375 ou SK-MEL-5 [44].

\section{Anticorps bispécifiques dans la conception de nouveaux récepteurs chimériques d'antigènes (CAR)}

L'utilisation d'Abs dans la conception de récepteurs chimériques pour l'antigène (ou chimeric antigen receptor, CAR) est une avancée récente dans ce domaine. Un CAR est une protéine hybride simple chaine composée d'un domaine extracellulaire de liaison à l'antigène ciblé et dérivé d'un fragment d'anticorps fusionné à un espaceur, un domaine transmembranaire suivi d'une région intracellulaire de signalisation combinant un domaine d'activation $(\operatorname{CD} 3 \zeta)$ et un domaine de co-stimulation (CD28 ou 4-1BB [45]). Ces protéines sont ensuite exprimées dans des LT soit de donneurs sains (thérapie allogénique) soit de patients (thérapie autologue), le produit cellulaire recombinant ainsi obtenu étant ensuite injecté aux patients et représentant le produit thérapeutique [45]. Malgré l'extraordinaire efficacité clinique observée chez des patients atteints de leucémie aiguë lymphoblastique et de myélome multiple, un échappement au traitement est parfois observé. C'est principalement pour éviter ce type de rechute que sont aujourd'hui conçus et développés des CAR bispécifiques fondés sur un module de reconnaissance portant deux sites de liaison à deux antigènes tumoraux différents.

Un CAR ciblant à la fois les molécules des lymphocytes B CD19 et CD20 a été conçu comme un format associant deux fragments d'anticorps simple-chaîne fusionné par un espaceur de type glycine-sérine [46]. Ce produit est pleinement actif contre les cellules tumorales exprimant CD19 ou CD20 [46] et maintient son activité cytolytique même après perte d'expression de l'un des deux antigènes. Un autre CAR ciblant simultanément CD19 et CD22 a été conçu par l'équipe de Crystal Mackall, à Stanford. La structure du double module de liai- son est différente de celle décrite précédemment: les deux domaines Fv sont contenus dans une structure en boucle de type [VL1-VL2-VH2-VHI] liés par des espaceurs appropriés ${ }^{13}$.

Des charpentes non-Ig ont également été utilisées pour construire le module de liaison de CAR, notamment des DARPIN. Ainsi la fusion d'un fragment d'anticorps simple-chaîne dirigé contre l'EGFR et d'un DARPIN ciblant HER2/neu a permis de construire un module de liaison bispécifique, présentant une plus grande sélectivité pour le tissu tumoral par rapport aux tissus sains exprimant également l'un ou l'autre antigène [47].

\section{Conclusions}

Capitalisant à la fois sur le succès médical et commercial des inhibiteurs de PD-1/PD-L1 et CTLA-4, sur l'augmentation de nos connaissances sur la biologie et l'immunologie des tumeurs, ainsi que sur la conception de nouveaux formats d'anticorps et autres charpentes protéiques, une explosion de nouvelles molécules à visée thérapeutique a vu le jour au cours de ces dix dernières années, associée dans la plupart des cas à la création de jeunes pousses industrielles soutenues activement par des sociétés de capital-risque et des fonds d'investissements largement dotés.

Les Abs ont une place de choix dans ce nouvel arsenal thérapeutique en développement, tout particulièrement dans le domaine de l'immuno-oncologie. Ils permettent en effet, soit d'apporter des propriétés biologiques inédites à l'Abs, associées au co-engagement des deux cibles, soit d'améliorer le profil de sécurité par rapport à une combinaison d'anticorps, en restreignant sa localisation tissulaire, soit également de combiner en une seule molécule les activités de deux anticorps conventionnels, réduisant ainsi les coûts de développement clinique et de fabrication.

La majorité de ces produits sont encore à un stade de développement clinique précoce. Dans les années à venir, des données cliniques plus robustes permettront de faire émerger les meilleurs formats et associations de cibles et, ainsi, de mieux explorer le potentiel de cette classe émergente de nouvelles molécules thérapeutiques. $\diamond$

\section{SUMMARY}

Bispecific antibodies, novel therapeutic candidates harnessing the immune system

Over the past ten years, an increased knowledge of tumor biology and immunology allowed the design and

\footnotetext{
${ }^{13}$ Schultz et al., ASH 2018.
} 
development of novel therapeutic antibody and protein scaffold formats, where bispecific antibodies (Abs) play a major role. The latter molecules can (1) bring novel pharmacological properties through the co-engagement of two targets, (2) increase the safety profile as compared to a combination of two antibodies thanks to a targeted relocation to the tumor and (3) reduce development and manufacturing costs associated with single drug product. This review analyzes the different bispecific antibodies and scaffolds described in the field of immuno-oncology, their structure and major pharmacological and physico-chemical properties. $\diamond$

\section{LIENS D'INTÉRÊT}

Les auteurs déclarent n'avoir aucun lien d'intérêt concernant les données publiées dans cet article.

\section{RÉFÉRENCES}

1. Hirsch L, Zitvogel L, Eggermont A, Marabelle A. PD-Loma: a cancer entity with a shared sensitivity to the PD-1/PD-Ll pathway blockade. BrJ Cancer $2019 ; 120: 3-5$.

2. Vonderheide RH. The immune revolution: a case for priming, not checkpoint. Cancer Cell 2018 ; $33: 563-9$.

3. Brinkmann U, Kontermann RE. The making of bispecific antibodies. mAbs $2017 ; 9$ : 182-212.

4. Runcie K, Budman DR, John V, Seetharamu N. Bi-specific and tri-specific antibodies- the next big thing in solid tumor therapeutics. Mol Med $2018 ; 24: 50$.

5. Hober S, Lindbo S, Nilvebrant J. Bispecific applications of non-immunoglobulin scaffold binders. Methods $2019 ; 154: 143-52$.

6. Dreier T, Lorenczewski G, Brandl C, et al. Extremely potent, rapid and costimulation-independent cytotoxic $T$-cell response against lymphoma cells catalyzed by a single-chain bispecific antibody. Int J Cancer $2002 ; 100: 690-7$.

7. Topp MS, Kufer P, Gokbuget N, et al. Targeted therapy with the T-cell-engaging antibody blinatumomab of chemotherapy-refractory minimal residual disease in B-lineage acute lymphoblastic leukemia patients results in high response rate and prolonged leukemia-free survival. J Clin Oncol $2011 ; 29$ : 2493-8.

8. Goebeler ME, Bargou R. Blinatumomab: a CD19/CD3 bispecific T cell engager (BiTE) with unique anti-tumor efficacy. Leuk Lymphoma 2016 ; 57 : 1021-32.

9. Viardot A, Goebeler ME, Hess G, et al. Phase 2 study of the bispecific T-cell engager (BiTE) antibody blinatumomab in relapsed/refractory diffuse large B-cell lymphoma. Blood $2016 ; 127$ : 1410-6.

10. Bacac M, Colombetti S, Herter S, et al. CD20-TCB with obinutuzumab pretreatment as nextgeneration treatment of hematologic malignancies. Clin Cancer Res 2018 ; $24: 4785-97$.

11. Liu K, Zhu M, Huang Y, et al. CD123 and its potential clinical application in leukemias. Life Sci $2015 ; 122: 59-64$

12. Chu Sy, Pong $\varepsilon$, Chen $\mathrm{H}$, et al. Immunotherapy with long-lived anti-CD123 $\times$ anti-CD 3 bispecific antibodies stimulates potent T cell-mediated killing of human AML cell lines and of CD123+cells in monkeys: a potential therapy for acute myelogenous leukemia. Blood $2014 ; 124: 2316$.

13. Al-Hussaini M, Rettig MP, Ritchey JK, et al. Targeting CD123 in acute myeloid leukemia using a T-cell-directed dual-affinity retargeting platform. Blood 2016; 127 : 122-31.

14. Gaudet F, Nemeth JF, McDaid R, et al. Development of a CD123xCD3 bispecific antibody (JNJ63709178 ) for the treatment of acute myeloid leukemia (AML). Blood 2016; $128: 2824$.

15. Cho SF, Anderson KC, Tai YT. Targeting B cell maturation antigen (BCMA) in multiple myeloma: potential uses of BCMA-based immunotherapy. Front Imunol $2018 ; 9$ : 1821.

16. Hipp S, Deegen P, Wahl J, et al. BI 836909, a novel bispecific T cell engager for the treatment of multiple myeloma induces highly specific and efficacious lysis of multiple myeloma cells in vitro and shows anti-tumor activity in vivo. Blood $2015 ; 126: 2999$.

17. Pauza CD, Liou ML, Lahusen T, et al. gamma delta T cell therapy for cancer: it is good to be local. Front Immunol $2018 ; 9$ : 1305.

18. de Bruin RCG, Veluchamy JP, Lougheed SM, et al. A bispecific nanobody approach to leverage the potent and widely applicable tumor cytolytic capacity of Vgamma9Vdelta2-T cells. Oncoimmunology $2017 ; 7$ : el375641.

19. Bottcher JP, Bonavita $\varepsilon$, Chakravarty $P$, et al. NK Cells stimulate recruitment of $C D C l$ into the tumor microenvironment promoting cancer immune control. Cell $2018 ; 172$ : 1022-37 el4.

20. Reusch U, Burkhardt C, Fucek I, et al. A novel tetravalent bispecific TandAb (CD30/CD16A) efficiently recruits NK cells for the lysis of CD30+ tumor cells. mAbs $2014 ; 6: 728-39$.

21. Rothe A, Sasse S, Topp MS, et al. A phase 1 study of the bispecific anti-CD30/CD16A antibody construct AFM13 in patients with relapsed or refractory Hodgkin lymphoma. Blood 2015; 125 : 4024-31.

22. Gantke T, Reusch U, Kellner C, et al. AFM26-Targeting B cell maturation antigen (BCMA) for NK cell-mediated immunotherapy of multiple myeloma. Blood $2017 ; 130: 3082$.
23. Kerber A, Kluge M, Reusch U, et al. EGFR/CD16A tetravalent bispecific antibody AFM24 to engage NK-cells to kill EGFR expressing tumor cells and safety results in cynomolgus monkey studies. J Clin Oncol 2017 ; 35 : el4001-e.

24. Schmohl JU, Felices M, Oh F, et al. Engineering of anti-CD133 trispecific molecule capable of inducing NK expansion and driving antibody-dependent cell-mediated cytotoxicity. Cancer Res Treat 2017 ; 49 : 1140-52.

25. Gantke T, Weichel M, Herbrecht C, et al. Trispecific antibodies for CD16Adirected NK cell engagement and dual-targeting of tumor cells. PEDS 2017 ; $30: 673-84$.

26. Chan WK, Kang S, Youssef $Y$, et al. A CS1-NKG2D bispecific antibody collectively activates cytolytic immune cells against multiple myeloma. Cancer Immunol Res 2018 ; $6: 776-87$.

27. Hadad U, Thauland TJ, Martinez OM, et al. NKp46 clusters at the immune synapse and regulates NK cell polarization. Front Immunol $2015 ; 6: 495$

28. Ryan JM, Wasser JS, Adler AJ, Vella AT. Enhancing the safety of antibodybased immunomodulatory cancer therapy without compromising therapeutic benefit: can we have our cake and eat it too? Expert Opin Biol Ther $2016 ; 16: 655-74$

29. Ott PA, Hodi FS, Kaufman HL, et al. Combination immunotherapy: a road map. J Immunother Cancer $2017 ; 5: 16$.

30. Wozniak-Knopp G, Bartl S, Bauer A, et al. Introducing antigen-binding sites in structural loops of immunoglobulin constant domains: $\mathrm{Fc}$ fragments with engineered HER2/neu-binding sites and antibody properties. PEDS 2010 ; $23: 289-97$.

31. Everett KL, Kraman M, Wollerton FPG, et al. Generation of Fcabs targeting human and murine LAG-3 as building blocks for novel bispecific antibody therapeutics. Methods $2019 ; 154: 60-9$.

32. Wang J, Sanmamed MF, Datar I, et al. Fibrinogen-like protein 1 is a major immune inhibitory ligand of LAG-3. Cell 2019; 176 : 334-47 el2.

33. Das M, Zhu C, Kuchroo VK. Tim-3 and its role in regulating anti-tumor immunity. Immunol Rev 2017 ; 276 : 97-111.

34. Klein C, Schaefer W, Regula JT, et al. Engineering therapeutic bispecific antibodies using CrossMab technology. Methods 2019; $154: 21-31$.

35. Zalevsky J, Chamberlain AK, Horton HM, et al. Enhanced antibody half-life improves in vivo activity. Nat Biotechnol $2010 ; 28: 157-9$.

36. Moore GL, Bautista C, Pong $\varepsilon$, et al. A novel bispecific antibody format enables simultaneous bivalent and monovalent co-engagement of distinct target antigens. $m A$ Abs $2011 ; 3: 546-57$.

37. Chester C, Sanmamed MF, Wang J, Melero I. Immunotherapy targeting 4-1BB: mechanistic rationale, clinical results, and future strategies. Blood 2018; $131: 49-57$.

38. Giuroiu I, Weber J. Novel checkpoints and cosignaling molecules in cancer immunotherapy. Cancer) $2017 ; 23: 23-31$.

39. Rothe C, Skerra A. Anticalin((R)) proteins as therapeutic agents in human diseases. BioDrugs $2018 ; 32: 233-43$.

40. Barroso-Sousa R, Ott PA. Transformation of old concepts for a new era of cancer immunotherapy: cytokine therapy and cancer vaccines as combination partners of PD1/PD-L1 inhibitors. Curr Oncol Rep 2018; $21: 1$.

41. Travis MA, Sheppard D. TGF-beta activation and function in immunity. Annu Rev Immunol 2014 ; 32 : 51-82.

42. Knudson KM, Hicks KC, Luo X, et al. M7824, a novel bifunctional anti-PD-L1/ TGFbeta Trap fusion protein, promotes anti-tumor efficacy as monotherapy and in combination with vaccine. Oncoimmunology $2018 ; 7$ : e1426519.

43. Strauss J, Heery CR, Schlom J, et al. Phase I trial of M7824 (MSB0011359C), a bifunctional fusion protein targeting PD-Ll and TGFbeta, in advanced solid tumors. Clin Cancer Res 2018 ; 24 : 1287-95.

44. Ravi R, Noonan KA, Pham V, et al. Bifunctional immune checkpoint-targeted antibody-ligand traps that simultaneously disable TGFbeta enhance the efficacy of cancer immunotherapy. Nat Commun $2018 ; 9: 741$.

45. Lim WA, June $\mathrm{CH}$. The principles of engineering immune cells to treat cancer. Cell $2017 ; 168: 724-40$.

46. Zah $\varepsilon$, Lin My, Silva-Benedict A, et al. T Cells Expressing CD19/CD20 bispecific chimeric antigen receptors prevent antigen escape by malignant $B$ cells. Cancer Immunol Res $2016 ; 4$ : 498-508.

47. Srivastava S, Riddell SR. Engineering CAR-T cells: design concepts. Trends Immunol $2015 ; 36: 494-502$
TIRÉS À PART

T. Wurch 